\title{
ÉTICA PROFESIONAL COMO PROYECTO DE INVESTIGACIÓN
}

\author{
Professional ethics as a research project
}

\section{Éthique professionnelle comme sujet d'étude}

\author{
Ana Hirsch ADLER \\ Universidad Nacional Autónoma de México. Centro de Estudios sobre la Universidad. \\ Ciudad Universitaria, Edificio de la Unidad Bibliográfica, Centro Cultural \\ Universitario. 04510 México, Distrito Federal. Correo-e: hirsch@servidor.unam.mx
}

Fecha de recepción: diciembre de 2003

Fecha de aceptación definitiva: marzo de 2004

BIBLID [(1130-3743) 15, 2003, 235-258]

RESUMEN

El proyecto de investigación sobre ética profesional forma parte de un proyecto colectivo más amplio, que se está desarrollando en la Universidad Nacional Autónoma de México. Del marco teórico, para este artículo, se seleccionaron y exponen cuatro asuntos interrelacionados: razones de por qué la ética profesional constituye un tema relevante para las instituciones de educación superior, tres investigaciones sobre ética profesional realizadas en México, profesión y ética profesional y principios de la ética profesional.

Palabras clave: ética profesional, valores profesionales.

\section{SUMMARY}

The research project in professional ethics is part of a bigger and collective one, in process, in the National and Autonomous University of Mexico. From the frame of reference, this article refers to four aspects: some of the reasons that confirm that 
professional ethics is an important theme for higher education institutions, three research reports about professional ethics made in Mexico, professions and professional ethics and principles of professional ethics.

Key words: professional ethics, professional values.

SOMMAIRE

La recherche en matière d'éthique profesionnelle est l'une des parties les plus importantes et collectives, en processus, à l'Université National et Autonomous du Mexique. A partir du cadre mentionné, cet article référe à quatres aspects: quelques raisons qui confirment que l'éthique professionnelle est un sujet important abordé dans l'enseignement supérieur, trois rapports de recherche sur l'éthique professionnelle réalisés au Méxique, profession et l'éthique professionnelle et les principes de ces éthiques professionnelless.

Mots clef: éthique profesionnelle, valeurs professionnelles.

\section{INTRODUCCIÓN}

La investigación sobre ética profesional forma parte del proyecto colectivo Valores universitarios y profesionales de los estudiantes de posgrado de la Universidad Nacional Autónoma de México, financiado por el Programa de Apoyo a Proyectos de Investigación e Innovación Tecnológica de la UNAM. Los objetivos son: explorar valores científicos, de ética profesional y de ética cívica, en una muestra de grupos de alumnos y programas de posgrado de dicha universidad, por áreas de conocimiento y construir una propuesta de formación valoral.

Específicamente, en ética profesional se están llevando a cabo tres actividades complementarias: a) conformación del marco teórico, b) entrevistas a académicos en España y México y c) construcción de un cuestionario-escala para ser aplicado a grupos de estudiantes de posgrado de la Universidad de Valencia y de la Universidad Politécnica de Valencia y para ser replicado en la UNAM $^{1}$. A continuación, se menciona brevemente el proceso de investigación en estas tres tareas:

\subsection{Marco teórico}

El marco teórico se fue construyendo paulatinamente con informes de investigación, bibliografía, hemerografía, documentos y tesis de doctorado, principalmente de autores de México y España. También se analizaron algunos trabajos de

1. El cuestionario-escala es una propuesta del Dr. Juan Escámez Sánchez de la Universidad de Valencia. Su construcción y aplicación están asesoradas también por la Dra. Rafaela García López. 
investigadores del Reino Unido, Estados Unidos de América, Francia y Alemania y documentos de la Comunidad Europea en general.

La vía de acceso a la información fue directa en el tema de ética profesional y ética de las profesiones e indirecta, sobre: valores específicos, principalmente responsabilidad; ética profesional como parte de las éticas aplicadas; sociología de las profesiones, específicamente sobre ideología del profesionalismo e identidad profesional, ética de las ciencias y de la investigación científica, ética de la docencia y ética de la universidad.

Consta de los siguientes aspectos: investigaciones relevantes del estado del conocimiento elaborado en México sobre valores universitarios y profesionales y especialmente sobre ética profesional, ética profesional como parte de las éticas aplicadas; antecedentes, definiciones y caracterización de profesión en general y de ética profesional en particular, diferencia entre ética y deontología, principios de la ética profesional, influencias económicas, técnicas y organizacionales en el ejercicio profesional, valores fundamentales como son: competencia profesional y responsabilidad, identidad profesional, ética de la docencia, ética de las ciencias y de la investigación científica y propuestas de formación valoral en este campo.

\subsection{Entrevistas}

En el segundo semestre de 2003 se llevaron a cabo nueve entrevistas con profesores de algunas universidades españolas, acerca del tema de ética profesional. Los entrevistados son de las siguientes universidades: Universidad de Murcia, Universidad Politécnica de Valencia, Universidad Complutense de Madrid, Universidad Pontificia de Comillas en Madrid y Universidad de Santiago de Compostela. Se llevarán a cabo otras dos en la Universidad de Valencia en abril del 2004. Las preguntas básicas de la guía de entrevista son:

1) ¿Cuáles son los principales valores que promueve la Universidad?

2) ¿Cuáles son los principales valores profesionales que promueve la Universidad?

3) ¿Considera la ética profesional importante? ¿Por qué?

4) ¿Considera necesario que existan asignaturas acerca de ética profesional en todas las titulaciones universitarias?

5) ¿Qué necesitan saber los estudiantes universitarios acerca de ética profesional?

6) ¿Qué contenidos de ética profesional pueden ser de utilidad para los estudiantes universitarios?

7) ¿Qué principios y valores sustentan la ética profesional?

8) ¿Cómo relacionaría usted la ética profesional con la identidad de los estudiantes universitarios?

9) Consideraría importante la ética profesional para los estudiantes de posgrado? ¿Por qué? 


\subsection{Escala de actitudes}

La construcción de la escala de actitudes sobre ética profesional tiene como sustento la propuesta de investigación construida por el Dr. Juan Escámez Sánchez, con base en la Teoría de la Acción Razonada de Fishbein y Ajzen (Escámez, 1998 y 1991).

El instrumento sobre ética profesional, con la asesoría de la Dra. Rafaela García López, de la Universidad de Valencia, se diseñó de la siguiente manera:

1. Se planteó la pregunta: En términos generales, indique los que a su juicio son los cinco rasgos más significativos de "ser un buen profesional".

2. Se aplicó a una muestra de 131 personas de cuatro sectores, en la ciudad de Valencia: profesionales ${ }^{2}$, profesores universitarios ${ }^{3}$, estudiantes de posgrado $^{4}$ y población abierta 5 . Además se indagó su ocupación, titulación, sexo y edad.

3. Con todas las respuestas y la frecuencia en que cada una de ellas fue nombrada se codificaron 29 rasgos.

4. Los rasgos se clasificaron en cinco tipos de competencias: cognitivas, técnicas, éticas, sociales y afectivo-emocionales.

5. Se construyeron 29 proposiciones que se enviaron a 10 jueces, considerados expertos en este campo en estudio: cinco profesores universitarios (de la Universidad de Valencia, de la Universidad Politécnica de Valencia y de la Universidad de Murcia) y cinco profesionales prestigiosos de Medicina, Leyes, Ingeniería, Filosofía y Educación, de la ciudad de Valencia.

6. Con los resultados de estas actividades, se elaboraran 118 proposiciones, clasificadas por los rasgos predominantes (seleccionados como tales por las personas de la muestra y por los jueces), las cinco competencias antes mencionadas y si responden propiamente a actitudes ${ }^{6}$, creencias ${ }^{7}$ o normas subjetivas $^{8}$.

2. En esa muestra hay 47 profesionales de las siguientes titulaciones: Periodismo, Derecho, Educación, Medicina, Arquitectura, Ingenierías, Ciencias de la Información y Enfermería.

3. Se trata de 21 profesores universitarios, que estudiaron: Psicología, Educación, Filosofía, Teología, Filología Inglesa, Finanzas Internacionales, Química, Derecho, Ciencias Económicas y Administrativas, Ingeniería Agronómica, Ingeniería Industrial e Ingeniería Química.

4. Los estudiantes de posgrado de esta muestra son 35 y estudian: Administración, Medicina, Comercio Internacional, Educación, Ingeniería y Arquitectura.

5. La muestra de población abierta es de 28 personas, que realizan funciones administrativas y técnicas en trabajos de administración, seguridad, limpieza y labores secretariales.

6. "Actitud es la evaluación favorable o desfavorable de los resultados de la conducta en cuanto afectan al propio sujeto. El componente afectivo-evaluativo es considerado como la característica específica de la actitud que la distinguiría de los otros conceptos del modelo. La evaluación favorable o desfavorable se concreta en una proposición hacia la realización o no de la conducta" (EsCÁmEz, 1991, 528).

7. "Creencia es la categoría que subsume toda la información que el sujeto tiene sobre el objeto de la actitud. (EscámEz, 1991, 528).

8. "La norma subjetiva se origina en la percepción del sujeto de que otras personas o instituciones, importantes para él, piensan que realizará o no una conductan (EsCÁMEZ, 1991, 528). 
7. Con las 118 proposiciones se llevó a cabo la prueba piloto del instrumento, que se aplicó a 50 personas: estudiantes de posgrado, profesores universitarios y profesionales. Para cada proposición se les pidió que optaran por una de cinco posibles respuestas (muy en desacuerdo, parcialmente en desacuerdo, ni a favor ni en contra, parcialmente de acuerdo y muy de acuerdo).

8. Los resultados se pasaron a una hoja de cálculo (del Programa SPSS) y se obtuvieron estadísticamente diversos análisis de fiabilidad, que permitieron reducir el número de proposiciones a 56.

9. Se elaboró la versión final de la escala de actitudes. Se pretende aplicarla a una muestra de grupos de posgrado de la Universidad de Valencia y de la Universidad Politécnica de Valencia en el primer semestre del 2004. Se realizará después en la Universidad Nacional Autónoma de México.

En el presente artículo se exponen algunas ideas que provienen del marco teórico sobre ética profesional: razones de por qué la ética profesional constituye un tema relevante para las instituciones de educación superior, tres investigaciones sobre ética profesional realizadas en México, profesión y ética profesional y principios de la ética profesional. Se desarrollan a continuación.

2. LA ÉTICA PROFESIONAL CONSTITUYE UN TEMA RELEVANTE PARA LAS INSTITUCIONES DE EDUCACIÓN SUPERIOR

Las razones son diversas:

- Las instituciones educativas tienen significativas funciones sociales y culturales en la construcción de la sociedad y con respecto a los importantes cambios que se están produciendo en el mundo, sobre todo cuando buscan modos diversos de disminuir la inequitativa distribución de la riqueza, promover la movilidad social y estudiar y formular opciones de solución para problemas prioritarios. Los valores y el comportamiento ético son parte de estos asuntos.

- Las profesiones y los profesionales, de todas las áreas del conocimiento, ocupan un lugar significativo en el mundo social, pues aportan bienes y servicios que requiere la propia sociedad. Su desarrollo y actuación están siempre en la mira de los sectores, grupos e individuos (a nivel local, regional, nacional e internacional). El comportamiento ético es parte intrínseca de la profesión y del sentido y proyectos de vida de los sujetos. Constituye, además, junto con la competencia profesional y técnica, lo que las personas mejor pueden apreciar de su labor.

- Hoy se vive una especial sensibilidad y demanda social de ética con respecto a los profesionales. Se insiste con mayor frecuencia en la importancia de incorporar elementos éticos en su formación y en el ámbito de 
investigación científica y sociocultural. Paulatinamente se han ido introduciendo asignaturas de ética y deontología profesional ${ }^{9}$ en las titulaciones universitarias y en las instituciones de educación superior y en los países de Europa Occidental y en los Estados Unidos de América y Canadá se han multiplicado los comités de ética, principalmente en los ámbitos de la ciencia. Han aparecido recientemente en sectores muy diversos, como son: universidades, empresas, ministerios y organismos, a escala nacional e internacional.

- Se hace referencia, en muchos discursos y propuestas, a la necesidad de que la universidad cambie, no para adaptarse mecánicamente a los lineamientos de las agencias internacionales, sino en el reconocimiento de las nuevas necesidades, estructuras y discursos que aparecen desde finales del siglo xx a nivel mundial y que marcan la situación de inicios de este siglo. En esta transformación, la formación en valores y el aprendizaje ético son una opción significativa.

- Se multiplican los conflictos éticos en el ejercicio profesional; entre otras razones porque se han desarrollado (o han sido aceptadas como tales) nuevas profesiones, se han generado campos de frontera interdisciplinarios y los profesionales se incorporan cada vez al trabajo en instituciones públicas y privadas. Esta incorporación puede limitar su independencia y su capacidad de tomar las decisiones más importantes, incluyendo las de carácter ético. Se suma a esto la crítica en los casos de comportamiento inmoral de los profesionales, tanto cuando actúan por cuenta propia, como con respecto a los que forman parte de las diversas organizaciones.

- La formación ética es una necesidad inaplazable en las universidades, tanto a nivel de las propias instituciones, como de todos sus actores. El papel socializador de las universidades en esta tarea sigue siendo crucial. No basta con preparar buenos profesionales, en conocimientos y habilidades en ciencia, tecnología y cultura, sino que incluye la reflexión de principios y valores. En las disciplinas científicas hay, en general, un mayor énfasis en la preparación cognoscitiva y técnica que en la formación ética. Sin embargo, esta última, añade consistencia moral al contenido científico y técnico y a las propias disciplinas.

- La ética, en y desde las universidades, es una oportunidad para la consolidación intelectual y moral de la vida universitaria y de la sociedad en su conjunto.

- La universidad ha sido, desde sus orígenes, la encargada de formar profesionales y especialistas en las diversas áreas del conocimiento. Además de

9. Augusto HorTAL (2002) considera que el objetivo fundamental de la asignatura de ética profesional dentro de un currículo universitario es proporcionar conocimientos y métodos básicos para hacerse cargo de la dimensión moral del ejercicio profesional. 
realizar esta importante tarea, puede contribuir decididamente en la formación de los ciudadanos.

- El conocimiento ha sido siempre la base de conformación de las profesiones ${ }^{10}$. La complejidad creciente del conocimiento, técnicas avanzadas y habilidades especializadas, así como de los problemas vinculados a su puesta en práctica, han generado mayor atención con respecto a la ética profesional.

- La ética profesional es un campo interdisciplinario, que puede contribuir a aminorar el aislamiento en que se encuentran las especialidades, para integrarlas en una perspectiva de conjunto. La ética compete a todas las profesiones, no sólo a cada una de ellas, sino también a los campos de interacción que se producen para resolver problemas sociales complejos.

- Ayuda a reflexionar sobre qué debe hacer un buen profesional para serlo. Para cada una de las profesiones, es significativo: clarificar los bienes y servicios que brindan a la sociedad, sus beneficiarios directos e indirectos, los modos más apropiados de ofrecer dichos bienes y servicios, la complejidad del trabajo profesional en las diversas organizaciones públicas y privadas, la posibilidad de que se produzcan conflictos éticos durante el ejercicio profesional, la problemática de identidad profesional cuando los estudios no coinciden con las ofertas de empleo, la existencia y vigencia de asociaciones, colegios profesionales y códigos y la oportunidad de contribuir - a través de la profesión - al mejoramiento de la sociedad. Estos conocimientos e información son de gran utilidad para los profesionales en ejercicio y para los profesores y estudiantes universitarios.

- En este campo de investigación, es relevante formular la pregunta acerca de los principios y valores prioritarios que conforman la ética profesional en las distintas áreas de conocimiento de las instituciones de educación superior. Especialmente importante es indagar si los alumnos los conocen y comparten y cómo piensan que pueden utilizarse en la práctica profesional. Complementariamente, saber si existen códigos éticos de la profesión y si los estudiantes universitarios pueden identificarse con ellos.

- La ética profesional es un tema privilegiado para promover la autoestima personal y colectiva de los estudiantes universitarios y de los profesionales, la calidad profesional y humana de lo que hacen y la estima social del servicio que prestan a la sociedad. Deben estar conscientes del servicio que ofrecen a la sociedad y del valor que representa.

10. Para Svensson (2003) la sociología de las profesiones está estrechamente vinculada a la sociología del conocimiento. 


\section{InVESTIGACIONES SOBRe ÉTICA PROFESIONAL EN MÉXICO}

La construcción del estado de conocimiento sobre valores universitarios y profesionales en México ha tenido recientemente tres fases. La primera es la coordinación del libro colectivo Educación y Valores (Hirsch, 2001), en tres volúmenes. En el segundo se compilaron diversas investigaciones sobre los valores universitarios y profesionales.

En un segundo momento, el Consejo Mexicano de Investigación Educativa (COMIE), organizó en México, por segunda ocasión, la elaboración de estados de conocimiento sobre una enorme gama de temas educativos. Tenían por objeto localizar y analizar las investigaciones realizadas en el país de 1990 a 2002. El trabajo se realizó en comisiones, que desarrollaron su labor entre 2001 y 2002. En el 2003 se publicó en forma de libros.

Uno de los grupos conformó la Comisión: Educación, Valores y Derechos Humanos, que subdividió el campo en estudio en: formación valoral en educación básica, formación ciudadana, aspectos filosóficos y teóricos, educación y valores de los mexicanos, derechos humanos y valores universitarios y profesionales. Específicamente, en el equipo sobre Valores Universitarios y Profesionales, de la Comisión, se localizaron ${ }^{11}$ y analizaron 53 reportes de investigación. Se clasificaron en ocho rubros: valores universitarios, valores profesionales ${ }^{12}$, ética profesional, valores de los estudiantes universitarios, valores de los profesores universitarios, valores psicológicos de los estudiantes universitarios y valores en el posgrado. En ética profesional se localizaron pocas investigaciones, pero algunas de ellas son muy relevantes y han tenido repercusión en instituciones educativas de México.

La tercera fase consiste en una actividad a largo plazo. La construcción del estado de conocimiento es una tarea permanente, por lo que se siguen recuperando informes de investigación sobre este campo temático.

A continuación se presentan tres investigaciones relevantes sobre ética profesional realizadas en México en estas tres etapas.

\subsection{Formación universitaria y compromiso social de los egresados}

En el primer caso está el trabajo "Formación universitaria y compromiso social: algunas evidencias derivadas de la investigación" de Carlos Muñoz Izquierdo, Maura Rubio Almonacid, Joaquina Palomar Lever y Alejandro Márquez Jiménez (2001), de la Universidad Iberoamericana (UIA) Ciudad de México, que sintetiza los resultados de tres investigaciones interrelacionadas sobre los valores de los egresados

11. En forma de libros, capítulos de libros, artículos en revistas especializadas, memorias extensas de congresos y tesis de maestría y doctorado.

12. Cuatro de ellos se refieren a valores en profesiones específicas: Psicología, Agronomía, Enfermería y Ecología. 
universitarios, en cuanto a su compromiso para contribuir a solucionar los problemas de los sectores socialmente desfavorecidos. Dos de ellas se realizaron únicamente en la UIA y la otra, comprende cinco instituciones de educación superior: tres públicas $^{13}$ y dos privadas ${ }^{14}$.

En el primer estudio, se aplicó un cuestionario a 706 egresados entre 1981 y 1991. En general, los resultados muestran una orientación individualista, contraria a la formación que se busca generar por parte de la universidad.

En el estudio comparativo entre las cinco universidades, la muestra fue de 754 egresados entre 1992 y 1994 . A ellos, les fueron planteadas preguntas similares a las que se hicieron a los egresados de la UIA; es decir, a qué fines destinarían su tiempo libre y sus recursos económicos adicionales en el caso de que se encontraran en dos situaciones hipotéticas: duplicar su tiempo libre sin reducir sus ingresos y duplicar sus ingresos sin aumentar su jornada laboral. Los resultados también confirman que la preocupación por los problemas de los sectores socialmente desfavorecidos ocupa un lugar secundario entre los intereses de los egresados.

La tercera investigación se propuso conocer las circunstancias del proceso y los agentes más significativos de socialización de los egresados. Se entrevistó a una muestra de 52 egresados de la UIA, que forman parte del conjunto de 706 egresados de la misma institución en los que se basó el primero de los estudios presentados. Para integrarla, se seleccionaron los sujetos que de acuerdo con su perfil valoral se ubicaban en los dos extremos de un continuo valoral; es decir los que tuvieron un perfil más centrado en sí mismos (individualismo) y aquellos cuyo perfil se centró más en el servicio hacia los demás (solidarismo). La mayoría de las respuestas en las entrevistas se centra primero en cualidades de las personas y segundo en objetivos familiares. El estudio concluye en que es relativamente baja la proporción de cualidades que reflejen algún interés en contribuir a solucionar los problemas que afectan a los sectores mayoritarios de la sociedad.

El marco teórico, las estrategias metodológicas y los resultados de este inmenso trabajo son muy importantes, lo mismo que las ideas que presentan los autores para promover la formación valoral universitaria. El estudio (que combina las tres investigaciones mencionadas) ha sido retomado por investigadores y estudiantes de posgrado en sus tesis en otras unidades de la UIA y por otras universidades privadas. La estrategia puede ser utilizada con éxito en todo tipo de instituciones educativas de educación superior.

13. Universidad Nacional Autónoma de México, Universidad Autónoma Metropolitana y Universidad Autónoma del Estado de México.

14. Instituto Tecnológico y de Estudios Superiores de Monterrey en el Estado de México y Universidad Iberoamericana, Ciudad de México. 


\subsection{Valores éticos de los psicólogos mexicanos}

La investigación intitulada Los valores éticos que promueven los psicólogos mexicanos en el ejercicio de su profesión (Pérez, 1999; Lafarga, Pérez y Schlüter, 2001) tuvo un carácter pionero en México. El estudio es relevante para entender esa profesión y proporciona ideas, conceptos, códigos profesionales y una herramienta de recopilación de información de gran utilidad para aproximarse al conocimiento de otras disciplinas; especialmente en sus aspectos éticos.

Los objetivos fueron: conocer y describir los valores éticos que los psicólogos mexicanos dicen promover en el ejercicio de su profesión y proporcionar a los posibles encargados de redactar un código ético, elementos de información de primera mano sobre los valores que más se requieren. Se aplicó un cuestionario a 553 psicólogos, con un nivel mínimo de licenciatura y al menos 6 meses de experiencia profesional. Se llevó a cabo por la Universidad Iberoamericana de la Ciudad de México y el Consejo Nacional para la Enseñanza e Investigación en Psicología de México.

Se revisaron los códigos éticos para los psicólogos de diferentes países. En ellos, se repiten aspectos significativos, como son: promover el bienestar de las personas a quienes sirven, mantener la competencia, proteger la confidencialidad o privacía, actuar responsablemente, abolir la explotación y defender la integridad de la profesión a través de una conducta ejemplar. Los resultados muestran que los valores más importantes para el ejercicio de la profesión, según el orden de importancia en que se mencionaron, son: respeto, responsabilidad, honestidad, capacidad profesional, confidencialidad, relaciones profesionales, investigación, ecología y justicia.

El cuestionario se basó en tres preguntas abiertas, que son importantes en la investigación de todas las profesiones. Éstas son: qué valores promueven en la práctica profesional, cómo describen cada uno de estos valores y cómo los jerarquizan en orden de importancia.

La investigación es de enorme utilidad, tanto por las preguntas abiertas que plantea, que resultaron exitosas en el trabajo de campo, como por el rastreo y análisis de los códigos profesionales de psicología en diversas partes del mundo. La propuesta del estudio puede ser utilizada con ventaja para estudiar otras profesiones a nivel nacional e internacional.

\subsection{Etbos profesional de los profesores que imparten la asignatura de Educación Cívica y Ética en la Secundaria en el Estado de Morelos, México}

María Teresa Yurén Camarena (2003) coordinó y desarrolló una investigación sobre la asignatura de Educación Cívica y Ética en la Secundaria en el Estado de Morelos. Esa materia de carácter obligatorio, creada en 1999, se imparte en todo el país.

El estudio se realizó entre abril de 2002 y enero de 2003. La información se reunió en 22 escuelas, con los siguientes procedimientos: entrevistas individuales a 44 estudiantes, 27 profesores y 11 directores, entrevistas a 16 grupos de alumnos 
y 96 observaciones. Se aplicó un cuestionario a 647 alumnos y 57 profesores en 20 escuelas secundarias, que se seleccionaron, combinando dos criterios: nivel socioeconómico (alto, medio y bajo) y eficiencia terminal (alta y baja).

De este enorme trabajo, aquí retomamos únicamente, algunos elementos sobre la ética de los docentes. La información en este tema se basa en las entrevistas abiertas a 27 docentes y la aplicación de cuestionarios a otros 57 . La construcción de seis categorías sobre la conducta de los profesores, resultó fructífera para organizar e interpretar los resultados del trabajo de campo y es de enorme utilidad teórica y metodológica para otras investigaciones vinculadas con este campo temático.

La autora, para las dos primeras categorias, retoma de Paulo Freire los estilos de instrucción. En la investigación se encontraron estilos en que predomina la "instrucción bancarian, que implica que el maestro ve al estudiante como una especie de vasija que debe ser llenada por el educador. Consiste básicamente en un proceso de transmisión y busca favorecer la memorización mecánica. La forma de instrucción privilegia: exposición del maestro, dictado, copia de textos, resúmenes, resolución de cuestionarios con base en el libro de texto y exposición de temas por parte de los alumnos. La segunda categoría es el comportamiento contrario, la unstrucción dialogante", que se basa en un diálogo alimentado por la acción y la reflexión. Favorece la actividad del estudiante con procesos de indagación, debate y construcción de explicaciones y contribuye a favorecer el aprendizaje significativo y la construcción de estructuras cognitivas.

Las cuatro categorías restantes se construyeron con base en la forma en que el profesor promueve la adquisición de saberes prácticos. En la de "socialización desapegada", el profesor está más interesado en cubrir los temas del programa que en la formación del estudiante y no muestra disposición para escucharlo y comprenderlo. Trabaja los contenidos conceptuales sin preocuparse de la dimensión de las actitudes y sin favorecer situaciones formativas que favorezcan la experiencia reflexiva y la participación del colectivo. La "Socialización inculcadora" consiste en que el profesor actúa como hablante pero no como escucha. No busca entender al estudiante ni convencerlo con razones, sino que impone sus pautas de valor, por el papel de autoridad que representa. En la "socialización pastoral", el profesor actúa como un hablante persuasivo que busca convencer al estudiante para que adopte ciertas pautas de valor. La última categoría se llama: "facilitación y acompañamienton. El profesor procura que el alumno adquiera, mediante la acción y la interacción dentro y fuera del aula, las herramientas de análisis, juicio y autorregulación que necesita. Favorece situaciones que demandan actitudes de acción, interacción y reflexión y se mantiene como interlocutor atento.

Estos seis tipos ideales no se presentan en forma pura en la realidad. Se encontraron en la investigación combinaciones diversas. Las más frecuentes fueron: a) bancario-desapegado, b) bancario-inculcador, c) bancario-pastoral y d) dialogantepastoral. El estilo dialogante-facilitador, que sería el más adecuado para esta asignatura se encontró limitadamente. 
La nueva asignatura de Formación Cívica y Ética en la educación secundaria creó tensiones para los profesores encargados de impartirla. En muchos casos, para disminuir el problema, los profesores optaron por enseñar nuevos contenidos con prácticas tradicionales, trabajando los saberes prácticos como si fueran hechos, conceptos o procedimientos por aprender. Es el caso del profesor que tiene un estilo "bancario-desapegado".

En los casos de estilos predominantemente "inculcador" o "pastoral" el proceso es distinto. Los maestros se preocupan por contribuir a que sus alumnos adquieran saberes prácticos, pero no a partir de la reflexión y la experiencia, sino a través de mecanismos de internalización de ciertas pautas de valor. Predomina el papel de guía o adoctrinador. Aunque hay resistencias para ello por parte de los alumnos, el peso del profesor y de toda la cultura escolar es tan fuerte, que aunque el programa de la asignatura busca discursivamente generar autonomía en los estudiantes, logra precisamente lo contrario.

Ninguno de los profesores en ejercicio tenía un perfil totalmente adecuado para la materia de formación cívica y ética, lo que los hizo sentir inseguros y rechazados por otros. Todos los profesores entrevistados hicieron referencia a dos valores que consideran centrales: confianza y respeto. También consideraron necesario rescatar y recuperar valores que consideran perdidos.

La autora concluye que la problemática que produjo la introducción de la nueva asignatura en la identidad de los docentes y el ambiente educativo poco propicio al cambio provocó que muchos de ellos retomaran el papel de instructores o adoctrinadores. Esta postura no permitió contribuir a satisfacer la formación de los estudiantes.

Las categorías y los resultados pueden ser aprovechados para indagar la situación de esta asignatura en las otras 31 entidades federativas de México.

Como puede verse, las tres investigaciones que aquí se presentan son diferentes entre sí, muestran la riqueza de investigación que se está produciendo en México en un campo temático relevante y aún poco explorado y aportan elementos significativos sobre la ética profesional.

4. Profesión y ÉTICA PROFESIONAL

\subsection{Profesión}

Para conceptuar la ética profesional es importante plantear qué se entiende por profesión. De las múltiples definiciones que hay, se retoman tres.

Para Adela Cortina profesión es:

Una actividad social cooperativa, cuya meta interna consiste en proporcionar a la sociedad un bien específico e indispensable para su supervivencia como sociedad humana, para lo cual se precisa el concurso de la comunidad de profesionales que como tales se identifican ante la sociedad (Cortina, 2000, 15). 


\section{Para Augusto Hortal, profesiones son:}

Aquellas actividades ocupacionales: a) en las que de forma institucionalizada se presta un servicio específico a la sociedad, b) por parte de un conjunto de personas (los profesionales) que se dedican a ella de forma estable, obteniendo de ellas su medio de vida, c) formando con los otros profesionales (colegas) un colectivo que obtiene o trata de obtener el control monopolístico sobre el ejercicio de la profesión y d) acceden a ella tras un largo proceso de capacitación teórica y práctica, de la cual depende la acreditación o licencia para ejercer dicha profesión (Hortal, 2002,51 ).

Juan Manuel Cobo considera que en el concepto moderno de profesión debe incluirse la ética. Por profesión se entiende:

Una actividad que ocupa de forma estable a un grupo de personas en la producción de bienes o servicios necesarios o convenientes para la sociedad (las profesiones entrañan una función social), con cuyo desempeño obtienen esas personas su forma de vida. Una actividad que se desarrolla mediante unos conocimientos teóricos y prácticos, competencias y destrezas propios de ella misma, que requieren una formación específica (inicial y continua), regulada por lo general social o legalmente y que deben utilizarse con ética profesional, esto es, con un uso adecuado..., responsable, respetuoso con los derechos humanos y acorde con la justicia (Cobo, 2003, 3).

Las definiciones de estos tres profesores universitarios españoles coinciden en elementos comunes, como son: se trata de una actividad social institucionalizada, las profesiones proporcionan bienes y servicios necesarios para la sociedad, se requiere de una formación especializada y reconocida para ejercerla y existen colectivos profesionales, que definen normas aceptables para el ejercicio de la profesión, generalmente a través de códigos éticos.

A las definiciones hay que agregar un hecho significativo, que consiste en reconocer que ninguna profesión es homogénea. La diversidad de campos y de personas en cada una de ellas permite comprender la riqueza de este campo en estudio. También hay que señalar que aunque las profesiones comparten elementos comunes y las pautas de cooperación son fundamentales, dentro y entre las profesiones se producen relaciones competitivas. Éstas se acentúan por la proliferación de profesiones, el creciente número de profesionales, el avance en los procesos de especialización y la formación de campos de frontera interdisciplinarios. La lucha por los espacios de actuación se vincula estrechamente con un tema importante como lo es el de identidad profesional.

Para Francisco Bermejo (2002), una profesión puede ser definida desde el punto de vista subjetivo, es decir la perspectiva de quien la practica, y objetivo, que se refiere al ámbito en donde se desarrollan sus actividades. En el primer caso, además de que le permite al profesional ganarse la vida, quienes la ejercen van transformando algunas de sus disposiciones personales y consolidando, a través de su 
trabajo, un nuevo modo de vinculación con la sociedad. Contribuye tanto a su maduración personal como a la construcción de la sociedad en la que vive. Realizar de manera satisfactoria o insatisfactoria el trabajo es decisivo para el mayor o menor éxito de nuestro plan global de vida. Además, el ingreso en una actividad y en una comunidad profesional dota al profesional de una peculiar identidad y sentido de pertenencia.

En el sentido objetivo se refiere a la necesidad de una larga preparación, para adquirir competencias, grados académicos y ciertos rasgos como son: identidad profesional, dedicación exclusiva, monopolio de la actividad profesional, reconocimiento social y autonomía profesional. Se presupone el continuo enriquecimiento de los saberes, habilidades y competencias.

Las profesiones tienen un carácter histórico y son cambiantes. Se han ido modificando sus metas, formas de ejercicio y relaciones entre colegas y con destinatarios de la actividad, principalmente por la generación de conocimientos y el aumento de la capacidad técnica y humana. Cada una de ellas busca el reconocimiento social, especialmente las disciplinas y áreas difusas. Se fortalecen algunas de las antiguas profesiones, surgen nuevas y se generan y desarrollan los campos interdisciplinarios.

\section{2. Ética profesional}

Para José Luis Fernández, la ética profesional es:

La indagación sistemática acerca del modo de mejorar cualitativamente y elevar el grado de humanización de la vida social e individual, mediante el ejercicio de la profesión. Entendida como el correcto desempeño de la propia actividad en el contexto social en que se desarrolla, debería ofrecer pautas concretas de actuación y valores que habrían de ser potenciados. En el ejercicio de su profesión, es donde el hombre encuentra los medios con que contribuir a elevar el grado de humanización de la vida personal y social (Fernández y Hortal, 1994, 91).

Con una visión menos centrada en el bienestar de la sociedad, pero que resalta fuertemente los valores, encontramos otra definición:

Conjunto de aquellas actitudes, normas éticas específicas y maneras de juzgar las conductas morales, que la caracteriza como grupo sociológico. Fomenta, tanto la adhesión de sus miembros a determinados valores éticos, como la conformación progresiva a una tradición valorativa de las conductas profesionalmente correctas. Es simultáneamente, el conjunto de las actitudes vividas por los profesionales y la tradición propia de interpretación de cuál es la forma correcta de comportarse en la relación profesional con las personas (Franca-Tarragó, en Pérez, 1999, 51).

Como se observa fácilmente, las definiciones acerca de profesión y las que se refieren de modo directo a la ética profesional están estrechamente articuladas. 
Freidson (2003) introduce una idea interesante, al afirmar que hay un ataque a la credibilidad de la ideología profesional. Considera que se produce con el fin de debilitar la voz de los profesionales que buscan influir en el cambio social, para evitar que tengan una opinión moral independiente al evaluar las políticas sociales. Los considera como una "tercera voz", frente al poder del Estado y del capital ${ }^{15}$. Para ello es necesario revitalizar las asociaciones profesionales, en contra del corporativismo y los problemas de mala actuación profesiona ${ }^{16}$.

Para este autor, las tres principales críticas que se hacen a las profesiones y a sus grupos organizados son por el monopolio que tiene cada una de las profesiones de ejercer socialmente un tipo de trabajo específico; el credencialismo, ya que la competencia profesional se acredita por medio de credenciales educativas especiales y el elitismo. Frente a estos cuestionamientos, considera que los colectivos profesionales que funcionan bien, organizan y hacen avanzar las disciplinas, mediante el control de la formación, de las acreditaciones y de la práctica. Afirma que el objetivo es asegurar y mantener la calidad del trabajo. El desarrollo de un cuerpo especializado de conocimientos y habilidades formales requiere de un grupo de personas con ideas afines que lo aprendan y practiquen, se identifiquen con él, lo distingan de otras disciplinas, se reconozcan como colegas en virtud de la formación común y de su experiencia con un conjunto similar de tareas, técnicas, conceptos y problemas laborales. Los grupos así formados son exclusivos y también inclusivos. El establecimiento de jurisdicciones exclusivas permite a los miembros concentrarse en ese marco común. El saber experto se basa en la investigación y en la acumulación de experiencia y los profesionales son depositarios de un conocimiento socialmente importante destinado a contribuir al bien público.

Los profesionales se sienten en la obligación de realizar su trabajo al máximo de sus competencias. Se presupone una identificación con las pautas ideales de la profesión y un alto grado de autocontrol de la conducta mediante un código ético interiorizado. Esto puede lograrse, principalmente, a través de un fuerte proceso de socialización en los valores de la profesión y en menor grado por el control externo ejercido por instituciones, asociaciones y colegios.

\section{PRINCIPIOS DE LA ÉTICA PROfESIONAL}

Para Augusto Hortal (2002), cada ética profesional genera, en su propio ámbito, una clasificación de situaciones, asuntos, conflictos y modos de abordarlos

15. Augusto HorTal (2002) y Adela CoRTINa (2000) coinciden en presentar a las profesiones y a los profesionales como una opción de la sociedad civil frente a esos dos poderes.

16. Al respecto MERTZMAN y MADSEN (1999) formulan las siguientes preguntas: ¿por qué las prácticas profesionales inadecuadas están tan extendidas?, ¿qué provoca que un profesional cruce la línea entre lo que está bien y lo que está mal?, ¿hay causas identificables de la mala conducta profesional? y ¿son responsables los individuos o los sistemas en que trabajan por estos problemas? 
y resolverlos, que permiten analizar lo que está en juego en la toma de decisiones. Los nuevos casos son juzgados, en primera instancia, con base en los elementos conocidos.

Los principios son imperativos de tipo general, que orientan acerca de lo que es bueno hacer y lo que debe evitarse. Se distinguen de las normas por ser más genéricos. Señalan grandes temas y valores de referencia, que hay que tomar en cuenta a la hora de decidir y de enfrentar casos problemáticos. Las normas aplican los principios a situaciones más o menos concretas.

Para este autor, los principios pueden ser el punto de partida o de llegada de una actuación. El "razonamiento moral descendente" va de los principios generales a otros más específicos, paulatinamente, hasta llegar a las decisiones singulares. Para poder ser aplicados, deben ser revisados e interpretados con respecto al contexto en que se producen y a las situaciones y casos que se busca resolver. $\mathrm{El}$ arazonamiento moral ascendente" parte de las actuaciones y decisiones singulares en situaciones concretas. De ahí se van generando criterios de actuación, hasta llegar al nivel más general de los principios. Ambos procesos se combinan.

En la ética profesional están implícitos al menos tres principios ${ }^{17}$.

\subsection{Beneficio o beneficencia}

La palabra beneficencia está compuesta de dos vocablos de origen latino, bene y facere, que podrían traducirse como hacer el bien. Hace referencia a la consecución de determinados bienes específicos de la práctica profesional correspondiente (Bermejo, 2002, 75).

Cada profesión se plantea y legitima frente a los demás la consecución de ciertos bienes y servicios. Para ser buenos profesionales, los individuos deben conocerlos y buscar su cumplimiento, tanto con respecto a los usuarios que reclaman un trabajo bien hecho, como de la sociedad en su conjunto, que pretende resolver problemas prioritarios con la contribución de los profesionales (Bermejo, 2002).

En este campo de investigación, lo primero que hay que plantearse es la finalidad de cada profesión. Se puede partir de generar y responder preguntas básicas como son: ¿qué bienes y/o servicios produce?, ¿para quién?, ¿de qué manera? En la evaluación de los profesionales, se consideran no sólo los directamente beneficiados por su actividad, sino también los individuos y grupos que se relacionan con las acciones desarrolladas.

17. Los autores los retoman fundamentalmente de la bioética. En ella se agrega un cuarto principio, el de "no maleficencia", que se refiere a tratar de evitar o al menos minimizar el daño que se causa a los sujetos individuales de la investigación, así como a otros individuos y grupos. Augusto HoRTAL (2002) lo integra dentro de los tres primeros principios. 
La ideología del profesionalismo (Freidson, 2003) enfatiza el uso del conocimiento y habilidades disciplinarias para el bien público. Aunque algunas disciplinas proporcionan directamente un bien específico a personas, grupos e instituciones, los bienes y servicios que se generan son siempre valorados con respecto a un bien común más amplio. Los profesionales y sus asociaciones tienen la obligación de valorar lo que hacen con esa perspectiva. Es evidente que para ello se requiere competencia profesional, que se adquiere por una formación inicial y continuamente actualizada de conocimientos y habilidades, de carácter teórico y práctico, para saber qué hacer y cómo hacerlo.

Augusto Hortal (2002) retoma de Alasdair MacIntyre la distinción entre bienes intrínsecos y extrínsecos. Los primeros están ligados a la adecuada realización de la práctica profesional y los segundos se refieren a las recompensas económicas, de poder y de prestigio que se asocian a ella. Es evidente que los bienes intrínsecos son los prioritarios y que se tergiversan las actividades profesionales cuando los esfuerzos están dirigidos únicamente al logro de beneficios personales.

Freidson (2003) considera que existe una larga tradición de estudiosos que defienden que los profesionales buscan el bien del cliente, del público o el desarrollo de una profesión, por encima de su propio interés económico. No puede haber una justificación ética para los profesionales que sólo buscan el beneficio personal, sobre la obligación de hacer un buen trabajo para el que lo necesite. El fortalecimiento de la legitimidad del profesionalismo requiere un claro reconocimiento de las implicaciones éticas del privilegio profesional y una fuerte resistencia a los acuerdos institucionales que enfatizan exclusivamente los incentivos económicos.

Detrás del secreto profesional (y de las patentes) se oculta, muchas veces, la apropiación y el monopolio sobre una parcela del conocimiento, que de ser manejada bajo principios éticos, ayudaría a resolver importantes problemas sociales.

Ejemplos de lo planteado hay muchos a nivel mundial. Uno de ellos, señalado por el Dr. Daniel Ramón Vidal ${ }^{18}$, catedrático de Tecnología de Alimentos de la Universidad de Valencia, consiste en la dificultad que hay de generar y distribuir alimentos básicos, desarrollados transgénicamente, a poblaciones y países que lo requieren con urgencia, debido a las patentes de las grandes compañias transnacionales. Lo mismo sucede con la producción de medicamentos que pueden combatir enfermedades contagiosas y pandémicas.

Aunque el principio de beneficio o beneficencia se plantea en general para todas las profesiones, es importante reflexionar en las diferencias que se producen entre ellas. Así, para cierto tipo de ciencias, como las exactas y naturales, podremos encontrar más fácilmente la reflexión sobre la ética profesional en la ética de

18. Conferencia en el curso ¿Guerra o desarrollo humano? (Desde una ética pública mundial), coordinado por la Dra. Adela Cortina, en la Universidad Internacional Menéndez Pelayo, Valencia, 7 a 11 de julio, 2003. 
la ciencia y de la investigación científica, mientras que en otro tipo de disciplinas, como las sociales y humanísticas y principalmente en aquellas que tienen una eminente labor asistencial, la relación directa con los beneficiarios de la actividad profesional ocupa un lugar predominante. Esto no exime, por supuesto, a ninguna profesión de la evaluación de las consecuencias que se producen por la toma de decisiones y el uso que se hace de sus resultados.

\subsection{Autonomía}

La palabra autonomía procede del griego: autos (sí mismo) y nomos (ley) y hace referencia a la capacidad que tiene cada cual de darse a sí mismo sus propias normas, procurando construir la propia vida a partir de ellas (Bermejo, 2002, 105).

En este segundo principio hay dos acepciones. Una de ellas se centra en el profesional, que requiere independencia y libertad para poder realizar adecuada y éticamente su trabajo y la otra se centra en el beneficiario, que posee derechos que deben ser respetados. Ambas posturas se plantean a continuación:

\subsubsection{Autonomía del profesional}

Se basa en el valor de la libertad (Etxeberria, 2002). Se refiere a la capacidad personal de tomar decisiones en el ejercicio de la profesión. Por este principio, se condena la presión extraprofesional, tanto de individuos, como de instituciones públicas y privadas en la toma de decisiones relevantes, que puede orillar a que se dejen de lado los comportamientos éticos.

Lo más importante de la ideología profesional (Freidson, 2003) es que está vinculada a valores trascendentes que le dan sentido y justifican su independencia. Los profesionales reclaman el derecho de evaluar las peticiones de empleadores o patrones y las leyes del Estado. Su revisión está basada en razones profesionales, que llevan a la convicción de que se está tergiversando el valor o propósito fundamental de una profesión. Los profesionales tienen que ser capaces de equilibrar el bien público con las necesidades más inmediatas de los clientes y empleadores.

\subsubsection{Autonomía del beneficiario}

En el segundo caso, que es la propuesta de Augusto Hortal (2002) y del grupo que trabaja este tema de manera sistemática en los Centros Universitarios de la Compañía de Jesús en España, el principio de autonomía busca corregir la falta de simetría entre quien ofrece el servicio y el beneficiario de la actividad.

El profesional por su preparación, acreditación y dedicación tiene un ascendente sobre sus clientes y usuarios. La desigualdad entre ambas partes puede producir abusos. Para evitarlos, es necesario que esté siempre en funcionamiento 
el principio de autonomía. Consiste en considerar que el receptor de los servicios (individual y colectivo) no es un ente pasivo, sino un sujeto protagonista. De ahí se deriva la obligación de garantizar a todos los individuos involucrados, el derecho de ser informados, de que se respeten sus derechos y de consentir antes de que se tomen decisiones con respecto a ellos; protegiendo de manera especial a los que no pueden decidir por sî mismos. "El usuario tiene el derecho y la obligación de colaborar en la resolución de sus problemas" (Bermejo, 2002, 105).

Cuando se respeta este principio, se establece una relación de carácter profesional, en la que se desarrollan ciertos acuerdos y estrategias conjuntas entre los profesionales y sus beneficiarios. En el caso de la universidad, por ejemplo, es necesario reconocer que los estudiantes pueden ejercer por sí mismos su autonomía, en plenitud de derechos, capacidades y responsabilidades.

Para Francisco Bermejo (2002) existen ciertos requisitos para que pueda darse una decisión autónoma. Son de dos tipos, los de carácter social y cultural, que implican que el contexto debe contar con condiciones propicias para ello y los de carácter personal, es decir, que los clientes y usuarios actúen con iniciativa y capacidad.

Sintetiza los requisitos en "querer", "saber" y "poder". En el primero, los clientes y usuarios deben contar con motivación para demandar al profesional el tipo de bienes y servicios que requieren. En el segundo, requieren de información, que incluye conocer otras opciones disponibles y las consecuencias que acarrea cada una de ellas. El tercero implica que si se quiere algo y se sabe cómo realizarlo, es necesario poder llevarlo a cabo. En todo proceso de decisión, el papel del profesional es apoyar, mediante sus recursos profesionales, la competencia e información de sus clientes y usuarios.

El autor retoma de Diego Gracia que la autonomía no es bipolar, sino un continuo entre dos extremos: la "acción completamente autónoma" y la "acción completamente no autónoman. Los dos polos no se dan en la realidad, así que puede aspirarse a que las decisiones sean "sustancialmente autónomas".

En algunas situaciones el principio de autonomía puede restringirse (Bermejo, 2002), aunque la decisión debe hacerla el profesional en cada caso. Los límites pueden suscitarse cuando: a) El usuario no tiene la competencia o los recursos personales para decidir, b) Puede producirse un daño grave para terceras personas, c) La conducta del usuario supone una infracción de la legalidad o una grave amenaza para las personas y las instituciones y d) El usuario se perjudica a sí mismo o sus decisiones no le benefician.

En relación con el consentimiento informado, Francisco Bermejo (2002) considera que el profesional debe obtener la autorización del usuario (individual y colectivo) para iniciar con él cualquier tipo de intervención, después de haberle explicado con claridad todas las condiciones. También hay circunstancias que producen excepciones a esa regla. Éstas son: falta de responsabilidad del cliente, posibles daños a terceros y limitaciones derivadas de la incapacidad del cliente (por dificultades de comprensión, falta de capacidad o falta de libertad). 
Loewenberg y Dolgoff (en Bermejo, 2002) señalan que el consentimiento informado incluye tres criterios básicos: conocimiento, voluntad y competencia. Sobre el primero, una persona puede ser considerada como suficientemente informada para dar su consentimiento si conoce lo que ocurrirá durante la intervención, lo que sucederá si no da su consentimiento y las posibles opciones alternativas. La voluntad y la competencia se limitan en el caso de niños y ancianos y en personas privadas de libertad o con capacidades mentales disminuidas.

\subsection{Justicia}

La ética profesional queda incompleta si no se enmarca en la perspectiva de una ética social, que permita entender en qué contribuye o puede contribuir el trabajo de cada profesión a mejorar la sociedad. Los profesionales son las personas y grupos más competentes y mejor ubicados socialmente para promover una distribución más racional y justa de los recursos, que son siempre escasos y que se requieren para conseguir múltiples y variados fines. Las preguntas básicas son: ¿Qué es lo justo? y ¿qué es prioritario cuando no hay recursos para satisfacer las demandas de todos?

Para Hortal (2002), este principio tiene que ver con:

1. El sentido social de la profesión. El colectivo profesional se hace responsable ante la sociedad de los bienes y servicios que busca promover. Se traduce en un compromiso a favor del bien público y con los problemas sociales que se refieren a temas del propio ámbito profesional. Los colectivos profesionales deben estar vinculados con las necesidades sociales.

2. El significado de los bienes y servicios que proporciona cada profesión en el contexto social en que se llevan a cabo, referidas al tema de la justicia, como son, por ejemplo, tareas de voluntariado y lucha contra la pobreza.

3. El desempeño profesional en espacios públicos y privados. Tiene que ver con el asunto de quién puede o no puede pagar por el servicio profesional que se requiere.

Un buen profesional tiene, o debería tener, siempre presente el contexto social de referencia y las obligaciones de justicia. La ética profesional permite reflexionar sobre si la función social que desempeña una profesión es la misma que la que la sociedad necesita de ella.

Con el principio de justicia (Bermejo, 2002) se hacen presenten tres protagonistas: los usuarios que reclaman determinados bienes y servicios, el profesional que requiere de medios para ofrecerlos y los responsables públicos, que representan al conjunto de la sociedad y buscan conseguir un cierto equilibrio entre las necesidades, exigencias y expectativas de todos. Es importante que los clientes y usuarios sean conscientes de que también dependen de la capacidad de las instituciones 
de responder a sus demandas y de su propia adaptabilidad a lo que éstas pueden proporcionar.

Freidson (2003) considera que sería apropiado declarar como profesionalmente inmorales las políticas sociales que nieguen un acceso igualitario a servicios tales como salud, educación y defensa jurídica. Afirma que deben ser juzgadas las instituciones en que ejercen profesionales que no cuentan con las condiciones de trabajo necesarias para realizar un correcto ejercicio. También es necesario cuando las instituciones sólo buscan maximizar sus ganancias, a costa de la calidad del trabajo y de limitar aún más la distribución de los beneficios.

\subsection{Otros principios}

Podríamos considerar los tres principios mencionados como los básicos. Hay

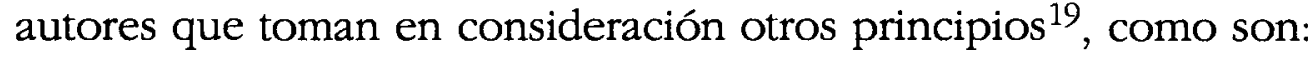

1. Evitar el daño. Consiste en no actuar de manera que se ponga en riesgo o se lastime a las personas. Equivale, en términos de los principios clásicos generados por la bioética, al principio de "no maleficencia". El evitar el daño a los hombres y a la naturaleza se vuelve muy importante, especialmente en el caso de las ciencias y la tecnología, que cuando se utilizan inadecuadamente tienen un enorme potencial destructivo. Para la inmensa mayoría de las personas, la ética de las ciencias se centra en la preocupación por los peligros del uso de la ciencia y la tecnología (no de éstas en sí) y por los límites que conviene establecer.

2. Fidelidad. El profesional hace promesas justas y cumple con sus acuerdos a aquellos a quienes presta el servicio. Es un derecho del cliente o usuario elegir al profesional y es un derecho de este último, aceptar o no la relación. Pero cuando ambas partes deciden iniciarla se entabla un acuerdo sobre la base de las expectativas previamente conocidas o formuladas. Los códigos conceden que hay una promesa explícita de cumplir el acuerdo.

3. Veracidad. Cuando se entabla la relación profesional-beneficiario, se establece un acuerdo implícito de que la comunicación se basará en la verdad.

4. Confidencialidad. Es el derecho que tiene cada persona de controlar la información referente a sí misma, cuando la comunica bajo la promesa —explícita o implícita- de que será mantenida en secreto. Se refiere a un criterio general de conducta que obliga al profesional a no discutir información acerca de los beneficiarios con otros. Obliga a guardar los secretos que uno conoce en razón del ejercicio profesional y a respetar la intimidad de las personas implicadas. En la práctica hay situaciones en que el profesional puede verse obligado a revelar, sin el consentimiento del cliente o usuario,

19. Corey, Corey y Callanan (PÉrez, 1999), Franca-Tarragó (PÉrez, 1999) y MERTZMAN y MadSex (1999). 
alguno de los detalles recibidos confidencialmente (Bermejo, 2002). Estos casos buscan: beneficiar de algún modo al cliente o protegerlo de algún mal que pudiera ocasionarse a sí mismo, proteger a terceros de algún perjuicio que pudiera ser ocasionado por parte del cliente, poner en común ciertos datos con otros colegas y profesionales y respetar la orden de alguna autoridad administrativa o judicial. El problema ético, en estos casos, radica en decidir acerca de la necesidad de contravenir el principio de confidencialidad. De todos modos, el usuario tiene derecho a que se le comunique, desde el inicio de la relación profesional, el tratamiento que se va a dar a la información, la obligatoriedad de la confidencialidad en general y las excepciones que pueden generarse. Todos los códigos deontológicos señalan la obligación que tienen los profesionales de mantener en secreto la información que han recibido con carácter confidencial. Si los beneficiarios no tienen esta seguridad no pueden expresarse con libertad. El profesional al garantizar la relación confidencial, manifiesta respeto por sus clientes y usuarios y por su libertad para tomar decisiones, incluyendo aquella de si quiere o no manifestar información públicamente.

5. Honestidad. Aunque este principio/valor se menciona escasamente, es importante para el correcto ejercicio profesional.

Juan Manuel Cobo (2003) propone unos principios éticos válidos para todas las profesiones. Unos provienen de la ética general, como son: dignidad, libertad, igualdad y derechos humanos, de los directamente beneficiados por el ejercicio profesional y de los indirectamente relacionados. Otros son propios de la ética profesional: beneficencia, autonomía, justicia, confidencialidad y responsabilidad profesional.

Acerca de los genetistas alemanes, Kerstin Wüestner (Hirsch y López, 2003) sintetiza los siete principios éticos centrales de la Sociedad para la Genética Humana: dignidad humana, derecho a la autodeterminación (la sociedad debe garantizar las condiciones básicas que permitan a las personas conocer todas las opciones y protegerlas de las desventajas económicas y sociales), igualdad, confidencialidad y secreto profesional, información completa, consentimiento informado y espontaneidad (la consulta y diagnóstico genético deben estar libres de toda presión).

Como puede verse, el tema de los principios de la ética profesional, es un asunto ineludible en la investigación de este campo. Son un punto de referencia, con los cuales contrastar el comportamiento real de los profesionales en sus lugares de trabajo y un elemento básico en la formación de los profesores y estudiantes universitarios. 


\section{BIBLIOGRAFÍA}

AltAREjOs, F. (1998) La docencia como profesión asistencial, en AltaRejOs, F; IBÁÑEz-MarTín, J. A.; JORDÁn, J. A. y JOVER, G. Ética docente. Elementos para una deontología profesional. Barcelona, Ariel, 19-50.

Bermejo, F. (2002) La ética del trabajo social. Bilbao, Desclée de Brouwer, S.A.

BIRD, S. (2003) Editorial. Ethics as a Core Competency in Science and Engineering, Science and Engineering Etbics, vol. 9, $\mathrm{n}^{\circ}$ 4, 443-444.

Сово, J. M. (2001) Etica profesional en ciencias bumanas y sociales. Madrid, Huerga Fierro Editores.

- (2003) Ética profesional, $4^{\circ}$ de pedagogia (curso 2003-04). Madrid, Universidad Pontificia de Comillas.

Comunidad EuRopea (2004) Proyecto Respect, Código para la conducta de la investigación social en la Unión Europea (http://www.respectproyect.org/code).

Cortina, A. (1997) Ciudadanos del mundo. Hacia una teoría de la ciudadanía. Madrid, Alianza.

- (2000) Presentación, el sentido de las profesiones, en Cortina, A. y Conil, J. 10 Palabras Clave en Ética de las Profesiones. Navarra, Editorial Verbo Divino, 13-28.

EsCÁmEZ, J. (1988) El marco teórico de las actitudes. I. El modelo de Fishbein y Ajzen, en EsCámez, J. y ORTEGA, P. La enseñanza de actitudes y valores. Valencia, Nau Llibres, 29-50.

- (1991) Actitudes en educación, en Altarejos, F.; Bouché, J.; Escámez, J.; Fullat, O.; Fermoso, P.; Gervilla, E.; Gil, R.; Ibáñez-Martín, J. A.; Marín, R.; Pérez, P. M. y Sacristán, D. Filosofía de la Educación Hoy, Conceptos. Autores. Temas. Madrid, Dykinson, 525-539.

EscÁmeZ, J. y GIL, R. (2001) La educación en la responsabilidad. Madrid, Ediciones Paidós Ibérica S.A.

ETXeBerRía, X. (2002) Ética de las profesiones. Bilbao, Desclée de Brouwer S.A.

EVETTS, J. (2003) Identidad, diversidad y segmentación profesional: el caso de Ingeniería, en SÁnChez, M.; Sáez, J. y Svensson, L. Sociología de las profesiones. Pasado, presente y futuro. Murcia, Diego Marín Librero-Editor, 141-154.

Fernández, J. y Hortal, A. (1994) Ética de las Profesiones. Madrid, Publicaciones de la Universidad Pontificia de Comillas.

FisHER, C. (2003) Developing a Code of Ethics for Academics. Commentary on Ethics for All: Differences across Scientific Society Codes. A Special Issue of Science and Engineering Etbics. The Role of Scientific Societies in Promoting Research Integrity, vol. 9, $\mathrm{n}^{\circ}$ 2, 171-179.

Freidson, E. (2003) El alma del profesionalismo, en Sánchez, M.; SÁEz, J. y Svensson, L. Sociologia de las profesiones. Pasado, presente y futuro. Murcia, Diego Marín Librero-Editor, 67-91.

Hirsch, A. (2001) Educación y Valores. México, Ediciones Gernika.

- (2002) Consideraciones teóricas acerca de la ética profesional, en LóPEZ, R. Educación y cultura global. Valores y nuevos enfoques en una sociedad compleja. México, Secretaría de Educación Pública y Cultura de Sinaloa y Universidad Autónoma de Sinaloa, 165-182.

- (2003) Elementos significativos de la ética profesional, en Hirsch, A. y López, R. Ética profesional e identidad institucional. México, Universidad Autónoma de Sinaloa, 27-42.

Hirsch, A.; Alcántara, A.; Gómez, G.; Herrera, A.; Ibarra, G.; Ríos, M. y Royo, I. (2003) Investigaciones de valores universitarios y profesionales, en BerTeLY, M. Comunicación, cultura y pedagogias emergentes. Educación, valores y derechos bumanos. México, Consejo Mexicano de Investigación Educativa, 1007-1032. 
HORTAL, A. (1994) La ética profesional en el contexto universitario. Lección inaugural del curso académico 1994-1995 de la Universidad Pontificia de Comillas. Madrid, Publicaciones de la Universidad Pontificia Comillas.

- (1995) La ética profesional en el contexto universitario, en Aula de ÉTICA. La ética en la universidad. Orientaciones básicas. Bilbao, Universidad de Deusto, 57-71.

- (2002) Ética General de las Profesiones. Bilbao, Desclée.

LAfarga, J.; PÉrez, I. y Shlüter, H. (2001) Valores éticos que promueven los psicólogos mexicanos en el ejercicio de su profesión, en Hirsch, A. Educación y Valores (II). México, Ediciones Gernika, 321-344.

Lozano, F. (2000) Pedagogía de la ética de la Ingeniería, Revista Educación y Pedagogía, vol. XII, $\mathrm{n}^{\circ} 28,59-67$.

- (2003) Ethical Responsibility in Engineering. A Fundamentation and Proposition of a Pedagogic Methodology. Valencia, International Conference on Engineering Education.

lozano, F.; Bon, A.; Siurana, C. y Calabuig, C. (2003) La educación en valores éticos en las enseñanzas científico técnicas. Experiencia del Grupo de innovación docente en educación en valores en los estudios científico-técnicos en la UPV. Valencia, Universidad Politécnica de Valencia.

Mertzman, R. y Madsen, P. (1999) Introduction to Professional Ethics, en Instituto Tecnológico Y DE ESTUDIOS SUPERIORES DE MONTERREY, Valores para el ejercicio profesional. México, Instituto Tecnológico y de Estudios Superiores de Monterrey, 119-126.

Mitcham, C. (2003) Co-Responsibility for Research Integrity, A Special Issue of Science and Engineering Ethics. The Role of Scientific Societies in Promoting Research Integrity, vol. 9, $\mathrm{n}^{\circ} 2,273-290$.

Muñoz, C.; Rubio, M.; PAlomar, J. y Márquez, A. (2001) Formación universitaria y compromiso social: algunas evidencias derivadas de la investigación, en $\mathrm{HIRSCH}$, A. Educación y Valores (I). México, Ediciones Gernika, 153-182.

NEWTON, L. (1999) Doing the Right Thing: the Recovery of Professional Conscience, en INsTITUTO TECNOLÓGICO Y DE ESTUDIOS SUPERIORES DE MONTERREY. Valores para el ejercicio profesional. México, Instituto Tecnológico y de Estudios Superiores de Monterrey, 208-217.

Núñez, I. (2000) Investigación, en Cortina, A. y Conill, J. 10 Palabras Clave en Ética de las Profesiones.Navarra, Editorial Verbo Divino, 153-173.

PÉREZ, I. (1999) Los valores éticos que promueven los psicólogos mexicanos en el ejercicio de su profesión. México, Universidad Iberoamericana.

Svensson, L. (2003) Introducción, en Sánchez, M.; SÁEZ, J. y Svensson, L. Sociología de las profesiones. Pasado, presente y futuro. Murcia, Diego Marín Librero-Editor, 13-28.

Tourouse, G. (2003) Mirada sobre la ética de las ciencias. Madrid, Ediciones del Laberinto.

WÜESTNER, K. (2003) Ética profesional. Los genetistas humanos en Alemania, en HIRSCH, A. y LÓPEZ, R. Ética profesional e identidad institucional. México, Universidad Autónoma de Sinaloa, 43-68.

YUREN, T. (2003) Tensiones identitarias y ethos profesional. El caso del profesor de formación cívica y ética en la escuela secundaria, en HiRSCH, A. y LóPEz, R. Ética profesional e identidad institucional. México, Universidad Autónoma de Sinaloa, 267-295.

Ziman, J. (2003) Non instrumental roles of science. Science and Engineering Etbics, vol. 9, $\mathrm{n}^{\circ} 1$, $17-27$. 